\title{
On a Class of Thermo-visco-plastic Constitutive Equations for Semi-solid Alloys
}

\author{
S. Benke ${ }^{a}$ and G. Laschet ${ }^{b}$ \\ ACCESS, RWTH Aachen, Intzestrasse 5, 52076 Aachen, Germany \\ as.benke@access.rwth-aachen.de, 'b.laschet@access.rwth-aachen.de
}

\begin{abstract}
Keywords: Theory of porous media, thermo-viscoplasticity, constitutive law, semi-solid behavior, equiaxed solidification, thixoforming

Abstract. The behavior of semi-solid alloys is quite different in tension, compression and shear and depends strongly on the morphology of the micro-structure. This article outlines a generalized viscoplastic material model for semi-solid alloys which reflects this complex viscoplastic behavior. From the generalized model a number of well known yield functions and viscoplastic material models for semi-solid and solid materials can be reproduces. The general model is applied to describe the behavior of the semi-solid A356 alloy below the coherency temperature during equiaxed solidification.
\end{abstract}

\section{Introduction}

The mechanical behavior of a semi-solid metal is influenced by the deformation of the solid and the flow of the remaining melt in the pore space. Both interact mechanically due to the exchange of momentum. Especially at high solid fractions and high strain rates a considerable liquid pressure will build up leading to a complex rate dependent constitutive behavior of the mush [1]. Experimental works on $\mathrm{Al}-\mathrm{Cu}$ [2] and $\mathrm{Sn}-\mathrm{Pb}$ alloys [3] show that the semi-solid coherent mushy zone presents significant differences in the deformation behavior under shear, compressive and tensile loading. Experimental investigations on globular semi-solid alloys used for thixoforming reveal a strong difference of the material response to shearing and uniaxial loading. Therefore, one of the major challenges in the simulation of semi-solid forming and solidification processes is the accurate rheological characterization and the appropriate constitutive modeling of this complex material behavior.

The morphology of the microstructure is quite different in thixoforming, rheocasting, equiaxed casting and direct chill casting processes. Indeed, in an equiaxed casting processes a coherent solid skeleton develops. In direct chill casting processes the dendrites grow preferable normal to the surface leading to a grain boundary covered with liquid, whereas in thixoforming processes the solid skeleton is composed of independent globular grains. The morphology of the solid microstructure determines to a large extend the response of the semi solid-alloy to different loading situations such as tension, compression and shear. Thus, for all mentioned processes the adopted yield surface of the coherent mushy zone has to take into account the different yield strengths in tension and compression. Thus, it must depend on the hydrostatic pressure explicitly.

The aim of this paper is the formulation of a constitutive model, enabling the simulation of the effect of isotropic and kinematic hardening, pressure dependence of yielding and the strengthdifference effect in tension and compression for a viscoplastic semi-solid alloy. The starting point for the formulation of the viscoplastic material model is the mathematical structure of a generalized yield condition in rate-independent elasto-plasticity introduced by Mahnken [4, 5].

\section{Theory of Porous Media}

The theory of porous media is the mixture theory restricted by the concept of volume fractions. The investigated semi-solid alloy is modeled as a saturated two-phase medium that consists of a solid 
dendrite network $\varphi^{s}$ and a viscous melt $\varphi^{l}$ in the pore space. In the volume fraction concept it is assumed that the solid dendrite network always models the control space and that the pores are statistically and homogenously distributed. This leads to a statistical homogenization of the body and enables a continuum mechanical description of the mixture. The volume fraction concept introduces the volume fraction $f^{\alpha}$ as scalar structural parameters relating the volume elements of the real phases $d v^{\alpha}$ to the volume of the control space $d v$ at the current position $\underline{x}$ at time $t$ :

$$
f^{\alpha}=f(\underline{x}, t)=\frac{d v^{\alpha}}{d v}=\frac{\rho^{\alpha}}{\rho^{\alpha R}},
$$

where $\rho^{\alpha}$ and $\rho^{\alpha R}$ are defined as the partial and real density. Neglecting the presence of pores in the mush, the saturation condition yields: $f^{s}+f^{l}=1$.

Assumptions In the current work we assume a material incompressible mixture body, where the density $\rho^{\alpha R}\left(T^{\alpha}\right)$ is a function of the temperature only. For isothermal conditions it follows that the volumetric deformation only results from a change of the pore space, i.e., from the volume fraction $f^{\alpha}$. Moreover, we assume a mass transition between liquid and solid due to solidification or melting:

$$
\hat{\rho}^{s}=-\hat{\rho}^{l}
$$

and take into account a local thermal equilibrium. Thus, the local temperature $T$ at the current position $\underline{x}$ in both constituents is equal: $T(\underline{x})=T^{s}=T^{l}$. At least, for the current study of the mechanical deformation behavior the chemical composition is supposed to be constant and equal in solid and liquid.

The model under consideration is now reduced to the independent but coupled fields: solid displacement $\underline{u}_{s}$, seepage velocity of the melt $\underline{v}_{l s}$, fluid pressure $p$ and the common temperature $T$ of the mixture. The corresponding equations for quasi-static deformation processes can be obtained from the kinematical relationships, the balance equations, the constitutive laws for the exchange of mass, momentum, energy and the constitutive material models as discussed in detail in [1]. The governing equations are namely the balance of momentum, mass, energy for the mixture and the Darcy law for the fluid flow.

\section{Constitutive Modeling}

From the evaluation of the entropy inequality of the saturated porous body where the saturation condition is considered as a constraint for the whole deformation process, we obtain the restrictions for the stresses $\sigma^{\alpha}$ and the exchange terms $[6,1]$.

Taking the mass exchange between liquid and solid due to the progressing solidification into account, the constitutive relations for the partial Cauchy stresses $\underline{\underline{\sigma}}^{\alpha}$ are given by:

$$
\begin{aligned}
& \underline{\underline{\sigma}}^{s}=-f^{s}\left(p+\rho^{s} \frac{\partial \psi^{s}}{\partial f_{s}}\right)\left(p+\rho^{s} \frac{\partial \psi^{s}}{\partial f_{s}}\right) \underline{\underline{I}}+{\underline{\underline{\sigma^{\prime}}}}^{s}, \\
& \underline{\underline{\sigma}}^{l}=-f^{l}\left(p+\rho^{l} \frac{\partial \psi^{l}}{\partial f_{l}}\right) \underline{\underline{I}},
\end{aligned}
$$

where $p$ denotes the fluid pressure, $\underline{\underline{I}}$ is the identity tensor, $\underline{\underline{\sigma}}^{s}$ is the effective stress in the solid dendrite network, $\psi^{\alpha}$ are the free Helmholtz energy functions of the constituents. 
Thermo-viscoplastic material law Assuming small elastic strains the total strain tensor of the solid $\underline{\underline{\varepsilon}}_{s}$ is defined as the sum of the thermal $\underline{\underline{\varepsilon}}_{s, t h}$, elastic $\underline{\underline{\varepsilon}}_{s . e l}$ and viscoplastic $\underline{\underline{\varepsilon}}_{s, t h}$ parts:

$$
\underline{\underline{\varepsilon}}_{s}=\underline{\underline{\varepsilon}}_{s, t h}+\underline{\underline{\varepsilon}}_{s, e l}+\underline{\underline{\varepsilon}}_{s, p p}
$$

The main ideas of the developed viscoplastic material model can be extended to finite deformation, but some care is required in selecting appropriate measures of deformation and stress.

The rate of the effective elastic stress of the solid is governed by the Hooke law:

$$
{\underline{\underline{\sigma^{\prime}}}}_{E}^{s}=\mathrm{H}\left(f^{s}, T\right) \underline{\underline{\varepsilon}}_{s, e l}
$$

where the elasticity tensor $\mathrm{H}$ depends on the solid fraction and on the temperature. The thermal expansion of a volume element of the solidified skeleton is given by:

$$
\underline{\underline{\varepsilon}}_{s, t h}=\alpha^{s}{ }_{T} \underline{\underline{I} \dot{T}} .
$$

During solidification the viscoplastic model has to describe the change of the microstructure form the coherent dendrite network to the solid body continuously with the help of macroscopic structural variables. The model should account for the plastic incompressibility of the material, thus dilatational flow should only occur if $f^{s}<1$ and for $f^{s}=1$ the classical von Mises plasticity and creep should be recovered. In general, a viscoplastic potential $\Omega$ for an elasto-viscoplastic continuum can be written in terms of the second-order stress tensor of the solid $\underline{\underline{\sigma}}^{s}$ and a tensor $\underline{\underline{S}}$ which accounts for some effects of anisotropy of the material behavior. For the modeling of kinematic hardening and strength difference effects $\underline{\underline{S}}$ can be reduced to a second order tensor which has the interpretation of a back-stress tensor. The viscoplastic potential can be simplified to:

$$
\Omega=\Omega\left(\mathrm{P}, \mathrm{I}_{1}(\underline{\underline{s}}), \mathrm{I}_{2}(\underline{\underline{s}}), \mathrm{I}_{3}(\underline{\underline{s}})\right) \text {. }
$$

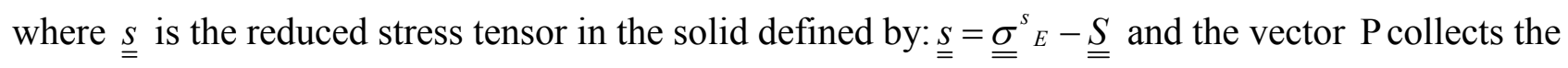
process variables such as fraction solid $f^{s}$, the temperature $T$, the equivalent viscoplastic strain $\varepsilon_{s, v p}^{e q}$ and other possible process variables. The three basic stress invariants $\mathrm{I}_{i}(\underline{\underline{s}})$ are defined as:

$$
\mathrm{I}_{i}(\underline{\underline{s}})=\frac{1}{i} \underline{\underline{1}}: \underline{\underline{s}}^{i},
$$

where $\prod_{=}^{1}$ defines a second order unit tensor. The additive decomposition of $s$ into its deviatoric and volumetric parts can be written as: $\underline{\underline{s}}=\underline{\underline{S}}^{\text {dev }}+\frac{1}{3} I_{1}(\underline{\underline{s}}) \underline{\underline{1}}$.

Relying on the ideas exploited by Mahnken [4, 5] for rate independent plasticity, we define a viscoplastic potential as follows:

$$
\begin{aligned}
& \Omega(\mathrm{P})=\frac{\eta(\mathrm{P})}{n+1}\left\langle\frac{I_{2}\left(\underline{\underline{s}}^{d e v}\right)+\aleph_{N}(\mathrm{P}) D(\mathrm{P})}{\eta(\mathrm{P})}\right\rangle^{n+1}, \text { where } \\
& \aleph(\mathrm{P})=\sum_{i=0}^{N} a_{i}(\mathrm{P}) I_{1}^{i}(\underline{\underline{s}}), \\
& D(\mathrm{P})=(1+\gamma(\mathrm{P}) \Gamma)^{-m_{D}},
\end{aligned}
$$




$$
\left.\Gamma(\mathrm{P})=I_{3}\left(\underline{\underline{s}}^{d e v}\right) / I_{2}{ }^{2 / 3} \underline{\underline{s}}^{d e v}\right)
$$

In relation (10) above the operator $\langle\cdot\rangle$ defines the Macauley brackets, equal to its argument if it is positive, or zero if it is negative. The parameter $n$ denotes the strain rate or Norton exponent which may be a function of the temperature and $\eta(\mathrm{P})$ is the viscoplastic consistency parameter. In expression (11) the parameter $a_{i}(\mathrm{P}), i=1 \ldots N$ and $\gamma(\mathrm{P})$ depend on the process variables, especially on the fraction solid $f^{s}$ and the cohesion of the solid network. The function $D(\mathrm{P})$ controls generally the deviation of the yield potential from a circle towards the compression and extension radii in the octahedral plane [4]. The viscoplastic strain-rate is given by an associated flow rule.

Remarks Using the mathematical structure of the relations $(10-13)$ we can reproduce a large number of viscoplastic potentials already used in the literature for the modeling of casting, sintering and thixoforming processes:

1. If one simplifies Eq. (12) towards $D=1$, and chooses $N=2, a_{0} \neq 0, a_{1}=0, a_{2} \neq 0, \underline{\underline{S}}=0$ the viscoplastic potential of Abouaf \& Chenot [7] for powder compaction can be reproduced. This model was used later by Nguyen et al. [8] for the modeling of semi-solid alloys in casting processes below the coherency temperature. By allowing a shift of the viscoplastic potential on the $I_{1}$ axis and thus introducing a hydrostatic back-stress the model was extended by Martin et al. [3]. Later, Martin et al. [9] and then Ludwig et al. [2] introduced an internal variable which measures the cohesion of the solid skeleton in order to take into account the interlocking of dendrites and their rearrangement on the micro-scale.

2. Kopp \& Horst [10] introduced a Drucker-Prager like yield function for the thixoforming of A356 alloys. The yield surface can be retrieved by setting $N=1, a_{0}=0, a_{1} \neq 0$, and using a slightly different expression of the deviation function: $D(\mathrm{P})=1+\gamma^{\prime} /\left(a_{1} I_{1}(\underline{\underline{s}})\right) \Gamma^{3}$.

3. The non-convex yield surface introduced by Kiuchi et al. [11] in order to model the remarkable drop of shear strength of the globular semi-solid mush in thixoforming processes can be retrieved by adopting $N=2, a_{0}=0, a_{1}=0, a_{2} \neq 0$ and the following definition of $D$ : $D(\mathrm{P})=1+\gamma^{\prime \prime} I_{2}^{2 / 3}\left(\underline{\underline{s}}^{d e v}\right) /\left(a_{2} I^{2}{ }_{1}(\underline{\underline{s}})\right)$.

4. For the case $N=2, a_{0} \neq 0, a_{1}=0, a_{2} \neq 0, \underline{\underline{S}}=0$ and $n=1$ the viscoplastic potential of Svoboda et al. [12] developed for powder compaction is obtained.

5. For the rate independent limit in Mahnken [4] it has been shown that by the use of a Taylor series for the cosh-function the yield function of Gurson [13] as well as yield functions of Tvergaard \& Needleman [14] and Rousselier [15] can be reproduced. This allows a smooth transition from the semi-solid state to the solid metal containing micro-pores. This is especially important for the modeling of heat treatment processes of cast parts which in general containing a small amount of shrinkage pores and gas pores after solidification.

6. Also for the rate independent limit the visco-plastic potential (10) reduces to certain wellknown yield criteria. For $D=1, N=0$ and $a_{0} \neq 0$ the von Mises plasticity is obtained.

\section{Application of the model to casting}

Based on the general formulation (10 - 13) we develop a rather simplified model for the modeling of coherent state of semi-solid alloys in casting processes. For this intended purpose the model must account for different behavior in tension, compression and shear and for the application in transient computer codes it should be computationally effective and the number of model parameters should be as low as possible. The following model is restricted to temperatures below the coherency temperature $T<T_{c}$ where the solid forms a more or less continuous dendrite network which is able to sustain tensile stresses. 
Here, $\aleph_{N}=\aleph_{2}$ is defined as a second order function:

$$
\aleph_{2}(\mathrm{P})=a_{0}(\mathrm{P})+a_{1}(\mathrm{P}) I_{1}(\underline{\underline{s}})+a_{2}(\mathrm{P}) I_{1}^{2}(\underline{\underline{s}}),
$$

The parameter $a_{0}$ describes the influence of strain hardening. Here, a combination of a saturation term and a linear hardening term will be used:

$$
a_{0}(\mathrm{P})=-\frac{1}{3}\left(Y_{0}+q\left(1-\exp \left(-b{\varepsilon_{s, v p}}^{e q}\right)+h \varepsilon_{s, v p}^{e q}\right)\right)^{2}\left(1-f^{s}\right)^{2}
$$

Here, $Y_{0}, q, b$ and $h$ are temperature dependent material parameter. The first and second order terms in Eq. (14) describe the deformation of the pore space of the dendrite network and thus reflect the internal cohesion of the solid dendrite network due to dendrite interlocking and the presence of liquid films. Both parameters $a_{1}, a_{2}$ are functions of the fraction solid $f^{s}$. The odd term is included in order to model the strength difference effect in tension and compression which is observed experimentally. In general, the evolution of the back-stress tensor $\underset{\underline{S}}{\dot{S}}$ is given by the Prager law. If the different behavior in tension and compression is modeled by the shift of the flow potential in the $I_{1}$ direction as done by Martin et al. [3], the following formulation of the back-stress can be used:

$$
\underline{\underline{S}}=c_{1} \dot{\underline{\varepsilon}}_{s, v p}-\left(f^{s}\right) \dot{f}^{s} \underline{\underline{I}}
$$

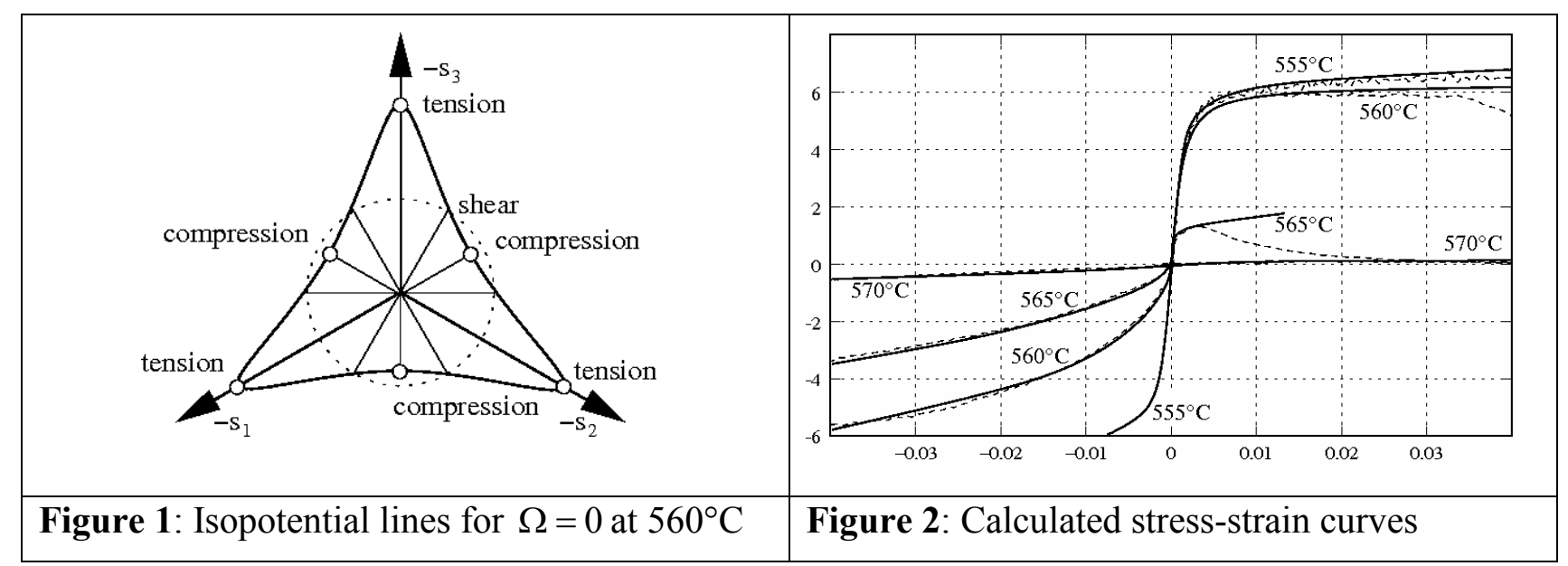

Based on the experimental results for uniaxial tensile and compressive tests the model parameters have been identified for an A356 alloy. The tests were conducted starting from the solid regime by first heating the solid as cast specimen with a rate of $1 \mathrm{~K} / \mathrm{s}$ to the defined testing temperature between the coherency temperature $585^{\circ} \mathrm{C}$ and the solidus temperature of $559^{\circ} \mathrm{C}$. Fig. 1 shows the isopotential line $\Omega=0$ at $560^{\circ} \mathrm{C}\left(f^{s}=0.99\right)$ in the octahedral stress plane. At this temperature the ratio between compression and tension is around 2.4. The experimental calculated uniaxial tension and compression curves for various temperatures are displayed in Fig. 2. The uniaxial tensile and compression tests can be reproduced very well until in tension at small strains a break-down of the load carrying capacity occurs. This is characterized by grain rearrangement and the loss of coherency of the dendrite network. 


\section{Conclusion}

In this paper a constitutive model has been formulated in order to simulate the effects of isotropic and kinematic hardening, and the strength difference in tension and compression for an elastoviscoplastic semi-solid material. The general model is based on the theory of porous media for a binary porous medium taking into account the mass exchange between solid and liquid due to the progressing solidification. The formulation of the constitutive model for the solid phase has its origins in the mathematical structure of a generalized plasticity model for the rate independent case as proposed by Mahnken [4, 5]. This approach was extended to rate dependent plasticity. Using this model several existing visco-plastic flow potentials from literature for semi-solid alloys can be retrieved. It seams that two power terms are sufficient in order to model the deformation behaviour. The model has been applied to simulate the experimental findings for a coherent semi-solid A356 alloy during equiaxed solidification. The proposed model is very general in order to describe the complex deformation behaviour of semi-solid alloys in casting and forming processes. Future work should be directed to the investigation of the functional relations between the model coefficients. As experimental investigations are very limited these studies will also be based on results of micromechanical modelling and on metallographic analyses.

Acknowledgments The authors thank the German Research Foundation (DFG) for the financial support within the joint research project PAK222: "Thermo-mechanical modelling and characterization of the solid-fluid interactions in casting processes".

\section{References}

[1] S. Benke and G. Laschet: Comp. Mat. Sci., in press, 2008

[2] O. Ludwig et al.: Mater. Sci. Eng. Vol. A413-A414 (2005), p. 174

[3] C.L. Martin, D. Favier and M. Suery: Int. J. Plast. Vol. 15 (1999), p. 981

[4] R. Mahnken: Comput. Methods Appl. Mech. Engng. Vol. 190 (2001), p. 5057

[5] R. Mahnken.: Int. J. Plast. Vol. 18 (2002), p. 801

[6] R. de Boer: Theory of Porous Media (Springer-Verlag, Berlin, 2000)

[7] M. Abouaf and J.L. Chenot: J. Th. and Appl. Mech. Vol.5 (1986), p. 121

[8] T.G. Nguyen, D. Favier and M. Suery: Int. J. Plast. Vol. 10 (1994), p. 663

[9] C.L. Martin, M. Braccini and M. Suery: Mater. Sci. Eng. Vol. A325 (2002), p. 292

[10] R. Kopp and M. Horst.: Proc. of the $7^{\text {th }}$ S2P Conference (2002), p. 719

[11]M. Kiuchi et al.: Annals of the CIRP Vol. 50 (2001), p. 157

[12] A. Svoboda et al.: Inter. J. Numer. Methods Eng. Vol. 43 (1998), p. 587

[13] Gurson, A. L.: J. Appl. Mech. (1977), p. 2

[14] Tvergaard, V. and Needleman, A.: Acta Metall., Vol.32 (1984), p. 157

[15] Rousselier, G.: Nucl. Eng. Design Vol.15 (1987), p. 97 